\title{
A researcher's perspective on electronic scholarly communication
}

\author{
Elaine Lally, Institute for Cultural Research, University of Western Sydney
}

\begin{abstract}
New information and communications technologies are transforming scholarly communication, just as they are transforming all areas of life. This paper gives a humanities and social sciences researcher's perspective on these changes. Scholarly communication has a profoundly contradictory nature. In an economy in which information is increasingly regarded as a highly tradeable and valuable commodity, scholarly work is a commercially valuable resource. Yet, from the point of view of its producers and consumers, it is a kind of 'gift' object, part of the circulation of discourse within their community. This profound paradox is, indeed, a clash of cultures, emerging out of irreconcilable contradictions between traditional values of scholarship and the commercialising processes of globalisation. Researchers and research intermediaries need to find new ways of working together in order to understand and take full advantage of the emerging forms and media for scholarly communication. From the point of view of researchers themselves, scholarly communication is still, as it has always been, a form of community-building and knowledge creation within a community of scholars. As researchers increasingly rely on new technologies to support and enhance their community building, the creation of new forms of scholarly community is enabled. The trend towards increasing interdisciplinarity across all disciplines is also accelerated, as researchers are able to access a wider range of materials than was available in traditional formats in localised libraries. New forms of electronic scholarly communication are also driving transformations in the quality control and accreditation processes for research information. With editorial peer review as the traditional means of accreditation, librarians and other information specialists have not been as involved as publishers within the process. This is changing as researchers are needing navigational tools for the information environment which distinguish between accredited and non-accredited information.
\end{abstract}

Keywords: electronic scholarly communication; humanities and social sciences research; electronic research communities; globalisation of information; research and technological change

\section{Scholarly traditions and technological change}

Life was undoubtedly simpler for academics in the 'good old days' of research practice, which many of us remain nostalgic for. Well-defined named disciplines gave definition to the range of literature that was relevant to any particular field of study (particularly helpful for postgraduate students and new entrants to the field) and it was clear where to go to find that literature: to the library. Disciplinary boundaries were policed through the efforts of members of the scholarly community themselves, often via scholarly societies as the publishers of the major journals in disciplines and sub-disciplines. Life 
was simpler for libraries, too, who could think in terms of 'holdings' for the particular disciplines of their constituencies. Scholars would simply visit the library to keep abreast of new developments in their field, and would have their own subscriptions to particularly important journals in that field.

The Internet has changed all this. Unsurprisingly, technological change has had the most rapid impact in the science-based disciplines, where academics are most comfortable with technology and are often actively at the forefront of developing technological means to support their research. Academics in the humanities and social sciences have undoubtedly been slower to take up the technology en mass, but now, with the exception of a very few diehard eccentrics, they have enthusiastically taken up at least the internet technologies of email and the world wide web and integrated them into their teaching and research (see Applebee et al. 2000 for a recent Australian survey). The ease of use and widespread acceptance of email and the web means that these two media have become the twin pillars of the infrastructure of the electronic research environment for the humanities and social sciences researcher. ${ }^{1}$

From the point of view of the academic as a consumer of research information in both traditional and electronic forms, there is a sense that it is not possible to keep up with the amount of material that is out there to be read. And even were it possible to keep up with the flood of relevant material, it would not necessarily be possible to find it all, since it's no longer clear where to look to find it. By and large, for academics themselves, this problem results in a vague sense of unease and ambivalence towards the technology - which seemingly promises so much but often delivers frustratingly little - rather than a sense that there is a looming crisis for scholarly communication.

However, the seemingly continuous acceleration of these complex and interrelated technological, social and cultural changes seems to induce a sense of vertigo in those of us who seek to follow, chart and interpret or respond to them. From the point of view of those knowledge intermediaries who support researchers and research infrastructure - including libraries, publishers and government agencies which fund research - there does seem to be a pervasive sense of crisis. For example, a recent conference on the impact of globalisation on Australian academic research and publishing (held in Sydney in July 2000 and hosted by the National Scholarly Communications Forum) was entitled 'Scholarship in peril?'

It is often said that there is a lack of detailed information about how researchers use electronic information which would lead to a better understanding of the needs of the users of scholarly information (NLA: 3). In this paper, I would like to give a researcher's perspective on the electronic research environment from the point of view of the interdisciplinary 'new humanities'. The aim of the paper is to bring together an overview of some of the ways in which the emergence of and increasing reliance on electronic forms of scholarly communication is changing the process of doing research. 


\section{Communication and community}

The initial point that I would like to make is that researchers are both readers and writers, and there is indeed little separation between the two activities as part of the overall process of doing research. Publishers are the traditional intermediaries who take care of the writing and editorial end of the process, whereas librarians have traditionally taken care of making the written products of the process accessible to readers. The process is not, however, one which begins with writing and ends with reading, which would perhaps be the publisher's perspective on it. Nor is it a process which begins with reading and ends with writing, as the library community might conceptualise it. The research process involves both reading and writing as intrinsic, inseparable and continuous aspects of the researcher's practice. This practice also necessarily involves interaction with other scholars throughout the process: work in progress is presented at conferences and subjected to the scrutiny and comment of other scholars. The peer-review and editorial processes are also important aspects of this communicative research context, since it is only through these activities that a piece of work is polished to the accepted professional standard.

Scholarly communication is indeed just that - communication - rather than a process within which scholarly products are produced and consumed. It often seems to researchers that knowledge intermediaries - including not just librarians and publishers but also our university administrators and funding agencies - view scholarly communication according to such a production/consumption paradigm. While the current preoccupation with the 'economics' of scholarly communication is undoubtedly useful from the point of view of all those intermediaries who are responsible for the funding of our knowledge infrastructure, I would suggest that in order to understand how the research process might best be supported it may also be useful to pay some attention to the changing characteristics of scholarly writing as a communications medium.

If communication processes, centrally, are what authors and readers want and need to be supported within the new electronic environment, then it is clear that, for them, this is more about the ongoing conversation within a group of scholars than it is about the production and consumption of scholarly 'information' or knowledge. Scholarly communication has clearly always been based on the ongoing interaction and discussion within a community of scholars. In the good old days the community of scholars was often identified with a learned society, but as researchers increasingly rely on new technologies to support and enhance their community building, both scholarly communication and scholarly communities are taking on new forms.

Internet technologies have continued the trend made possible by the telephone and the fax, for joint research to be conducted across great distances. It is now possible for collaborators to work together on a piece of writing, exchanging drafts by email. 
If traditionally scholars were grouped into named disciplines and associations based around a common interest, there is a 'postmodern' trend is for communities to be much more informal and transient. Email discussion lists spring up based on particular topics or narrow areas of focus. Tightly focussed conferences bringing together a critical mass of scholars working on particular issues can attract participants from all over the world, because of the ease with which conference announcements can be disseminated electronically. Scholarly communities may be based not around a particular abstract intellectual interest but be focused on concrete projects or activities, and may be quite ephemeral.

While email lists illustrate one model of a distributed scholarly community, another example is that of community building around a website. An example of the creation of a website as a deliberate attempt to build a community of scholars working in a particular area - in this case comparative ethnographies of internet use - is the website built to support Miller and Slater's book on the Internet in Trinidad (2000). The website (at http:/ / ethnonet.gold.ac.uk/) supports the book by providing extensive illustration of websites referred to in the text (including preserving their interactivity to a limited degree), but this site is also interesting as an attempt to extend the ways in which a scholarly publication is used by its target community. The site includes a guest book and discussion list, and the authors explictly are attempting to set up a process whereby the audience can engage directly in debate with each other and with the authors.

Johnson suggests that these new forms of community building may pose a threat to traditional knowledge intermediaries who do not adapt to the changing times:

Given that producers and consumers can now directly contact each other, only those intermediaries that add significant value are likely to survive. Libraries will need to place greater emphasis on the value they can add to the knowledge process. (1999: 57)

Johnson refers to this process as 'disintermediation'. While this term suggests that the relationship between scholars as producers and scholars as consumers is becoming less mediated, it is clear that in many respects the knowledge creation process has in fact become more mediated. However, the technologies involved serve to hide that mediation, masking it in a seeming transparency and immediacy. In the past, librarians and publishers were highly visible as mediators of the interaction between scholars, so it now appears that the technology may be making these roles redundant.

There is indeed a novel sense of being able to communicating directly within a global community of scholars. Because of this, it is no longer clear to scholars what services publishers and librarians are providing to them - it is as if the technology is doing all the work. As Johnson suggests, it is perhaps time for knowledge intermediaries to rethink their role and contribution to the processes of scholarly communication and community building. 


\section{Interdisciplinarity}

Research across all disciplines, but particularly in the humanities and social sciences, is increasingly interdisciplinary in character. New information technologies broaden the potential user base for all scholarly material that exists in an internet-accessible form. They therefore, potentially at least, make it possible for scholarly communication to take place between people who would not otherwise have come into contact with each other, and enable community to be founded on novel intellectual grounds.

A number of studies have shown that researchers generally access a wider range of serial titles when they are available digitally through aggregator services than was available in print form in their libraries (NLA: 4). This is clearly shown in the PEAK experiment at the University of Michigan (Bonn, Mackie-Mason et al. 1999) in the case of Science serials, although there is no reason not to suppose that these results would not be generalisable across disciplines. This kind of behaviour on the part of researchers is both a consequence of increasing interdisciplinarity, but the increase in accessibility of materials aggregated across disciplines is certainly one of the factors accelerating these changes. The traditional idea that it is possible to identify a core list of journals for a particular constituency of client researchers is increasingly untenable, particularly in new interdisciplinary niche areas (c.f. Joswick \& Stierman 1997).

Access to the knowledge archive is also particularly important for the humanities and social sciences. Indeed, retrodigitisation projects (digitising back issues of journals and canonical texts) may be a more important resource for these disciplines than for the sciences, where it is the latest information and results which researchers need to access (hence the rise in importance of preprint services and 'grey' literature). The humanities and social sciences remain heavily dependent on the 'canon' and continued use of 'classic' or particularly influential articles and texts. As Professor Malcolm Gilles, President of the Australian Academy of the Humanities pointed out in his presentation to the 'Australia's Information Future' workshop, a humanities book or article might only attract substantial interest a decade or more after publication (Gilles 1999: 82). Back issues of journals therefore remain of important research interest, and initiatives such as the JSTOR project (www.jstor.org), which involves the progressive digitisation of back runs of core journals, are therefore of crucial importance for humanities and social sciences scholars. This project encompasses more than 100 titles across a range of disciplines, including some in humanities and social sciences, but mainly in the traditional disciplinary areas such as anthropology and history, rather than in 'new humanities' disciplines such as cultural studies.

Electronic databases allow easy access to material based on keyword searches independently of the disciplinary niche of the journals being searched. Physical library collections and the traditional printed serial formal have also always allowed users to find similar content located together, through associative 
browsing. Developments in electronic information delivery, such as full-text article databases, tend to serve best the 'search' mode of information gathering, while some kinds of web-based information organisation facilitate browsing. Traditionally, researchers have relied heavily on being able to alternate between keyword searching and associative browsing as approaches to the literature. The development of web-based portals or gateways, bringing together heterogeneous but related material is an important developmental initiative, but there is a danger that the important issue of quality control will be lost in the race to put everything on the web.

The editorial processes of peer review are the traditional means of offering a vetting, gatekeeping and quality control function (through the initial selection of articles) and quality improvement (through refereeing and revision). This process ensures the production of research information which is accredited within a particular scholarly community. Publishers, in particular, are an important part of this process, but this is perhaps an area where librarians have not previously seen themselves as making a contribution. Librarians do, however, have an important role to play in the quality control processes in the new environment, and particularly in helping researchers find their way through the amorphous and seemingly infinite mass of research information that is 'out there' in cyberspace.

The technology is clearly already enabling, as we saw above, linkages and connectivity not just between pieces of information, but also between people. What researchers are increasingly finding useful are those electronic portals or gateways which give access to a heterogeneous range of information services. As Rowse suggests, researchers are saying 'Give me a place where I can find all kinds of things that may interest me' (Rowse 1999). Bringing together the diverse kinds of information which researchers draw in during the process of doing and disseminating research, including things which were never found in the library in the traditional sense, clearly makes sense in the electronic environment. This would include such things as conference announcements and calls for papers, job advertisements, email discussions or bulletin boards and preprint servers, as well as scholarly publications such as journals and monographs in electronic form.

Many university libraries are attempting to create portals themselves which provides a unified and user-friendly interface to information services of interest to their user communities, some of which are subscription based and hence not publicly available. There is also an increasing recognition on the part of governments and national research organisations that organised access to internet-based information is an issue of national information infrastructure. The UK's Resource Discovery Network (http:/ / www.rdn.ac.uk) is an important initiative in this area.

Publishers are clearly also realising that they need to rethink the value they add to the scholarly communication chain. The recently launched 'arena' portal programme of Taylor and Francis (see for example the Media and Cultural 
Studies Arena at http:/ /www.culturalstudiesarena.com/) is clearly a response to the challenge to add more value to their products.

At the moment the creation of these gateways is fragmented and ad-hoc, and it is not yet clear how these services will eventually be taken up and used by researchers, to the point where they are built integrally into their research practice. It is therefore not yet clear how these services can best meet the needs of their users.

From the point of view of researchers, it appears that some of the most popular of these gateways are those established by enthusiastic scholars. This activity perhaps illustrates new roles for scholars themselves in defining the development of the medium. Two such examples from the discipline of cultural studies are the CULTSTUD site (http:/ / www.cas.usf.edu/communication/rodman/cultstud/) and the PopCultures site (http:/ / www.popcultures.com/). These are very popular among a 'connected' group of cultural studies scholars. Indeed each of these sites - like Slater's site mentioned above - was developed and is maintained through the efforts of a single dedicated scholar. It is clear that such projects can be both an important way of developing a public profile for that individual within the community, but also acts informally as a way of filtering, authenticating and 'peer-reviewing' web-based material.

\section{Scholarly information: gift or commodity?}

Engaging in scholarly communication is a necessary part of becoming a member of a particular academic community. From the point of view of the researcher, the intellectual 'goods' which circulate within these networks operate, not according to the logic of the commercial transaction, but according to the logic of the gift.

The distinction between objects which are exchanged as gifts and those which are exchanged as commodities is well-established in the anthropological literature (Gregory 1982). 'Gift' exchange transactions entail an ongoing social relationship between the transactors, whereas in the commercial relationship there is no expectation of ongoing interdependence. Once the money has changed hands that is the end of the transaction, whereas the exchange of gifts cements social bonds and establishes or maintains an ongoing relationship. As Hyde puts it: 'when gifts circulate within a group, their commerce leaves a series of interconnected relationships in its wake, and a kind of decentralized cohesiveness emerges' (1979: xiv).

The essence of the gift is that, even when given to someone else, the object retains something of its associations with its originator, and the object establishes a link between them. Commoditisation, on the contrary, is a process through which objects are placed in a context in which they have been alienated from their producers, former users, or prior context, and in which they have exchange value (in Marx's terms). Paradigmatically, once the commodified 
object has been transferred to another person, the original owner has no enduring legal rights over the object.

Scholarly work is at the same time both intrinsically inalienable from its originator, yet legal ownership of it is able to be reassigned to another (and this is often a precondition of a publisher's agreement to publish the work). Licensing the right to reproduce the work in specified ways, rather than simple assignment of copyright, is clearly the form of transfer of rights which does most justice to the dual nature of scholarly products (and it is to be hoped that, in the long term, this will be the form of agreement which becomes dominant).

The 'gift' nature of the scholarly literature has many parallels with the open source movement in software development. Raymond contrasts the commercial world's 'cathedral' model of product development with the 'bazaar' model of the open source movement, where there is no centralised control and planning, and peer to peer evaluation is used to evolve and develop the content produced (Raymond 1999). Indeed, the internet in its early days may be said to have been the largest gift economy the world has known, although proprietory interests are rapidly changing both its culture, and the nature of its ownership from public good to private property (in parts, at least).

This is the conceptual dilemma, in my view, underlying the ongoing discussion around the 'freeing' of the scholarly literature (see various articles by Harnad at http://www.cogsci.soton.ac.uk/ harnad/, and the discussion at http://amsciforum.amsci.org/archives/september98-forum.html). 'Freeing' the literature is essentially about liberating the literature from the privatised control of the commodity form, rather than being about actually making it free of cost. Information online is never really free in this latter sense: even when there are no unit or marginal costs in accessing material, there are significant infrastructure costs, many of which are met institutionally.

Scholarly products therefore have a profoundly contradictory nature. From the point of view of their producers and consumers, they are part of the circulation of discourse within their community, a kind of 'gift' object (among others, such as the peer reviewing for which scholars receive no financial reward). Hence it still strikes some as 'bizarre that academics produce the materials which publishers then require them to pay to read' (Bekhradmia 1999: 18). For publishers, however, scholarly work is a commercially valuable resource, in an economy in which information is increasingly regarded as a highly tradeable and commercially valuable commodity.

This is a profound paradox emerging out of irreconcilable contradictions between long-standing traditions and values in research and scholarship and the processes of globalisation and commercialisation. It is, indeed, a clash of cultures - commercial culture colliding with scholarly culture.

\section{Competition or cooperation?}

It is clear that there will be no return to the good old days when all a researcher needed to do to find their research material was to visit the library. In order to 
both understand and take full advantage of the emerging forms and media for scholarly communication, what is clear is that researchers and research intermediaries need to find new ways of working together.

The technology is changing so rapidly that researchers are generally not aware of all the facilities they have available to them. There are clear examples of spectacular uptake in the research process of particular technological innovations, of which email is perhaps the most stunning example. However, technological innovations often require a process of introduction which enables the researcher to explore what the technology can do for them. This is essentially a process of testing the technology's ability to build itself into the user's pattern of everyday activity and routine. There is often resistance to trying out new facilities, even though once that resistance is overcome the user may become an evangelical 'convert'. There is therefore a need for ongoing outreach on the part of knowledge intermediaries - working closely and interactively with the scholarly community.

The e-prints initiative (http:/ /www.eprints.org), is significant in that it is an example of a development which is driven from within the scholarly community, but which has attracted the support of information infrastructure providers, including universities and organisations such as JISC. Similarly, the California Digital Library hosts the e-scholarship project (http:/ / escholarship.cdlib.org), which promotes scholar-led initiatives in scholarly communication and which hosts a number of scholarly communities and provides support and infrastructure to their electronic publications.

Researchers generally alternate between searching (when looking for something specific) and browsing ('cherry-picking') as approaches to the literature. Yet, many of the systems currently being developed as aids to the literature essentially relate to directed searching. There is a need for more attention to support for electronic browsing. For example, the Amazon.com database is an invaluable research resource - it covers a wider range of material than is held in any one library collection and it gives information about books that have not yet been published. It is also associatively indexed, so that the record for a book gives links to suggested others within the same field of interest: harking back to the days when a visit to the library's shelves found not just the specific material looked for but also other material which is located with it. ${ }^{2}$

As Johnson has suggested, disintermediation may mean that knowledge intermediaries need to be adding significant value in order to survive in the new environment. While it is true that it is now possible for scholars to interact directly with each other, it remains the case that this interaction is, in technological terms at least, more mediated than ever. Paradoxically, Internet technology appears to involve both increased mediation (in terms of technologies and institutional arrangements which mediate access to research resources) and decreased mediation (in terms of direct contact and networking between researchers and providers of journal content). The decreased mediation may, however, be illusory - being based on increasing transparency 
of that mediation (technological and institutional) - since from the point of view of the individual researcher it appears that knowledge intermediaries are becoming ever more and more organised and powerful.

A competitive relationship between these groups will have profound effects for those who are the original producers of, and the end consumers of, this information (see McLean 1999 for a discussion of the complex issues surrounding this restructuring process). It is particularly important, therefore, to maintain cooperative rather than competitive relationships between different elements in the chain. So, it is perhaps not helpful when it is suggested that there are 'zealots' within the library community who see publishers 'as a breed of dinosaur destined for extinction' (Bekhradmia 1999: 18). It remains the case, and will in all likelihood for the forseeable future, that getting one's work published in a reputable commercially published journal, or one's book published by a commercial publisher will be essential to academic progression.

Governments, particularly research funding bodies, are also increasingly becoming interested in what is happening in this area. There is clearly 'national interest' in the provision of research infrastructure, since it relates to issues of national competitiveness and development and will become increasingly important as we move towards the 'knowledge economy'. These, we can only hope, will be beneficial times for both knowledge workers and intermediaries.

\section{Acknowledgements}

I would like to thank Anita Crotty and Paul Evans, of the Program Committee for the Information Online 2001 conference (16-18 January 2001, Darling Harbour, Sydney), for encouraging me to prepare an earlier version of this paper, which was presented at the conference (http://www.csu.edu.au/special/online2001/.

\section{Notes}

1 While CD-ROMs and earlier Internet protocols (such as Telnet interfaces) were ubiquitous only three or four years ago, they are no longer part of the mainstream. This interface convergence is definitely, from the user's point of view, a good thing: the more seamless and transparent the electronic environment can become, the better. The requirement for browser plug-ins to view certain kinds of documents (even the widespread PDF format) is still an obstacle to some of my colleagues. The user would prefer not to have to think about the technological infrastructure at all.

2 It might be argued that other library databases do this too, but it may be that there are lessons to be learnt about the way that Amazon does this - it is not necessary to click through to another 'related material' option to get this information, it is directly accessible.

\section{References}

Applebee, Ann, Peter Clayton, Celina Pascoe \& Harry Bruce (2000) 'Australian academic use of the Internet: implications for university administrators', Internet Research: Electronic Networking Applications and Policy, 10(2): 141-9. 
Bekhradnia, Bahram (1999) 'The scholarly information crisis in the UK: the Follett Report and beyond', in Australia's Information Future: Innovation and Knowledge for the 21st Century, Proceedings of a workshop held 3-4 March 1999 at the Australian National University, Department of Education, Training and Youth Affairs: 13-24.

Bonn, Maria S., Mackie-Mason, Jeffrey K. et al. (1999) 'A Report on the PEAK Experiment: Usage and Economic Behaviour', D-LIB Magazine, 5(7/8), Jul/ Aug.

Gilles, Malcolm (1999) 'Research needs for the new millennium: issues for the humanities', in Australia's Information Future: Innovation and Knowledge for the 21st Century, Proceedings of a workshop held 3-4 March 1999 at the Australian National University, Department of Education, Training and Youth Affairs: 816.

Gregory, Chris (1982) Goods and Commodities. London: Academic Press.

Harnad, Stevan (1999) 'Free at Last: The Future of Peer-Reviewed Journals' DLib Magazine 5(12) http:/ / www.dlib.org/dlib/ december99/12harnad.html

Hyde, Lewis (1979) The Gift: Imagination and the Erotic Life of Property. New York: Vintage Books.

Johnson, Ron (1999) 'The challenge of the knowledge economy: managing knowledge', in Australia's Information Future: Innovation and Knowledge for the 21st Century, Proceedings of a workshop held 3-4 March 1999 at the Australian National University, Department of Education, Training and Youth Affairs: 518.

Joswick, K.E. \& Stierman, J.K. (1997) 'The core list mirage: a comparison of the journals frequently consulted by faculty and students', College and Research Libraries, 58(1): 48-55.

Miller, Daniel \& Don Slater (2000) The Internet: An Ethnographic Approach. Oxford: Berg.

McLean, Neil (1999) 'Collaborating to compete: the search for new alliances', http:// www.lib.mq.edu.au/conference/mclean/collaborate/index.html.

National Library of Australia (2000) A Review of Studies of Users of Scholarly Information, report prepared for the Coalition for Innovation in Scholarly Communication, May.

Raymond, Eric S. (1999) The Cathedral and the Bazaar: Musings on Linux and Open Source by an Accidental Revolutionary, O'Reilly \& Associates. Also available online at http://www.tuxedo.org/ esr/writings/cathedral_bazaar.

Rowse, Mark (1999) 'Tell me what you want, what you really, really want!: Research into the online information needs of the STM journal user', http:/ / www.ingenta.com/ingentainstitute.htm. 
Biographical note: Elaine Lally is Executive Officer at the Institute for Cultural Research at the University of Western Sydney. Her research interests centre on new technologies and everyday life: The Computer at Home, a book based on her PhD research (a study of home computer ownership) is forthcoming with Berg. She is a member of the Editorial Committee of Communal/Plural: Journal of Transnational \& Crosscultural Studies (published by Taylor \& Francis).

\section{Contact details}

Dr Elaine Lally

Executive Officer, Institute for Cultural Research

University of Western Sydney

Locked Bag 1797, Penrith South DC NSW 1797 AUSTRALIA

Phone: +61 296859911

Fax: +61296859964

Email: e.lally@uws.edu.au 